\title{
Post-transcriptional Boolean computation by combining aptazymes controlling mRNA translation initiation and tRNA activation
}

\author{
Benedikt Klauser, $\dagger^{a}$ Athanasios Saragliadis, $\dagger^{a}$ Simon Ausländer, $\ddagger^{a}$ \\ Markus Wieland, $\ddagger^{a}$ Michael R. Berthold ${ }^{b c}$ and Jörg S. Hartig ${ }^{* a c}$
}

Received 13th March 2012, Accepted 1st June 2012

DOI: $10.1039 / \mathrm{c} 2 \mathrm{mb25091h}$

In cellular systems environmental and metabolic signals are integrated for the conditional control of gene expression. On the other hand, artificial manipulation of gene expression is of high interest for metabolic and genetic engineering. Especially the reprogramming of gene expression patterns to orchestrate cellular responses in a predictable fashion is considered to be of great importance. Here we introduce a highly modular RNA-based system for performing Boolean logic computation at a post-transcriptional level in Escherichia coli. We have previously shown that artificial riboswitches can be constructed by utilizing ligand-dependent Hammerhead ribozymes (aptazymes). Employing RNA self-cleavage as the expression platform-mechanism of an artificial riboswitch has the advantage that it can be applied to control several classes of RNAs such as mRNAs, tRNAs, and rRNAs. Due to the highly modular and orthogonal nature of these switches it is possible to combine aptazyme regulation of activating a suppressor tRNA with the regulation of mRNA translation initiation. The different RNA classes can be controlled individually by using distinct aptamers for individual RNA switches. Boolean logic devices are assembled by combining such switches in order to act on the expression of a single mRNA. In order to demonstrate the high modularity, a series of two-input Boolean logic operators were constructed. For this purpose, we expanded our aptazyme toolbox with switches comprising novel behaviours with respect to the small molecule triggers thiamine pyrophosphate (TPP) and theophylline. Then, individual switches were combined to yield AND, NOR, and ANDNOT gates. This study demonstrates that post-transcriptional aptazyme-based switches represent versatile tools for engineering advanced genetic devices and circuits without the need for regulatory protein cofactors.

\section{Introduction}

Manipulating naturally occurring and implementing artificial genetic circuits are of great importance for future diagnostic, therapeutic, and biosensing applications. ${ }^{1}$ Until today, molecular and synthetic biologists have assembled addressable genetic devices in order to implement oscillators, ${ }^{2}$ counters, ${ }^{3}$ signal filters, ${ }^{4}$ memory devices, ${ }^{5}$ photo-inducible switches ${ }^{6}$ and molecular computation. $^{7-9}$ Nevertheless, most of the currently available platforms for regulating gene expression utilize and hence strongly rely on the presence of proteins such as transcription factors.

\footnotetext{
${ }^{a}$ Department of Chemistry, University of Konstanz,

Universitätsstr. 10, 78457 Konstanz, Germany.

E-mail: joerg.hartig@uni-konstanz.de;Tel: +497531884575

${ }^{b}$ Department of Computer and Information Science,

University of Konstanz, Germany

${ }^{c}$ Konstanz Research School Chemical Biology (KoRS-CB),

University of Konstanz, Universitätsstr. 10, 78457 Konstanz, Germany

$\dagger$ Both authors contributed equally.

$\ddagger$ Present address: Department of Biosystems Science and Engineering,

ETH Zurich, Mattenstrasse 26, CH-4058 Basel, Switzerland.
}

However, post-transcriptional devices for controlling gene expression represent versatile systems that exploit interesting RNA properties such as catalysis and ligand-binding encoded in small sequence spaces compared to proteins..$^{7,8,10-13}$

A trademark of any computing device is the conversion of an input into a defined output signal, which in turn can be picked up by another sensor unit within an information processing cascade. The capacity of a computing device rests upon the scalability of its single subunits. Importantly, a processing unit can transform a single input into multiple outputs and vice versa. Any computational operation can be abstracted into binary Boolean logic gates. For illustration, binary logic gates are often represented by truth tables in which the performance for every possible combination of input states is shown. In Fig. 1A the truth tables of the AND, NOR, and ANDNOT gates are shown. Binary Boolean logic operations allow for the construction of highly complex information processing networks through the modular assembly of basic logic gates. The enormous potential of logic computation 


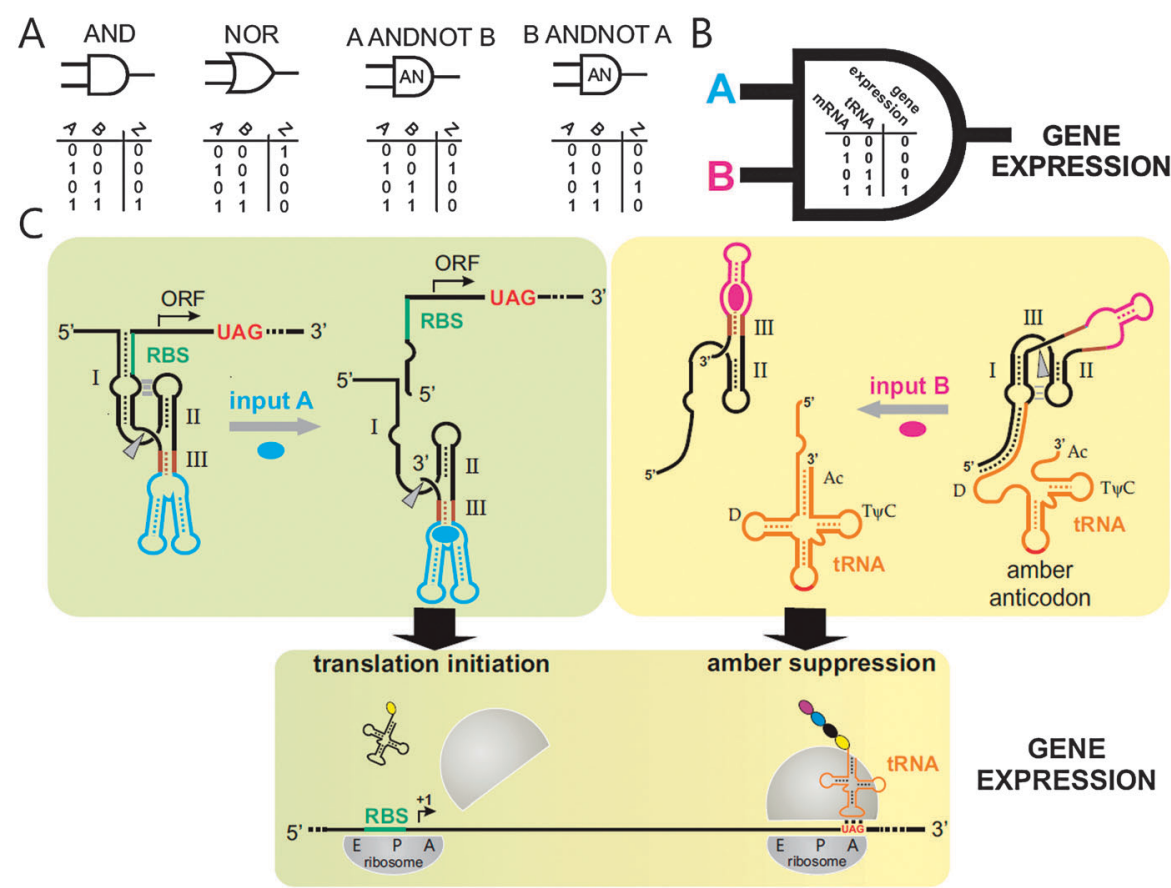

Fig. 1 Schematic illustration of two-input logic gates based on combinations of aptazymes. (A) Logic diagrams and truth tables for AND, NOR, and ANDNOT gates are shown. (B) The assembly of two HHRs controlling liberation of the RBS and regulating activation of an amber suppressor tRNA ${ }_{\text {CUA }}^{\text {Ser }}$ corresponds to an AND gate since both aptazymes are required to cleave for activating translation of the eGFP mRNA. Ligand-dependent regulation of the HHRs implements an additional layer of regulation allowing the construction of AND, NOR and ANDNOT logic gates. (C) Detailed illustration of the mechanisms of the aptazyme-based switches and their assembly to control a single eGFP transcript. The inputs are integrated via two aptameric sensor domains with diverging ligand specificities (inputs A and B). Hence, ligand addition controls eGFP expression via both mRNA translation initiation and tRNA activation.

has been demonstrated by the breath-taking advances in electronic engineering in the past decades. ${ }^{14,15}$

With respect to biomolecular computing, nucleic acids are particularly versatile for reading, writing, computing and storing information. In particular, researchers have made use of the diverse functions of DNA and RNA to construct simple information processors which are able to perform rudimentary molecular computation in vitro, ${ }^{16}$ whereas only a few artificial in vivo systems have been realized. ${ }^{7,8}$ Notably, the discovery of naturally occurring riboswitches has revealed a wide-spread class of cis-regulatory RNA control mechanisms. ${ }^{17}$ Riboswitches are located within the $5^{\prime}$-untranslated regions of an mRNA. Binding of a small molecule ligand to its aptamer domain controls gene expression. ${ }^{18}$ In addition to simple riboswitch architectures, aptamer domains assembled in tandem are able to control important cellular functions in a more complex, computational fashion. ${ }^{19-21}$ Win and Smolke reported a comparable artificial concept in Saccharomyces cerevisiae. They assembled cis-regulatory RNA-devices resulting in AND, NOR and NAND biomolecular devices. ${ }^{8}$ The basic concept in this and our own RNA-based switches of gene expression is a small molecule-dependent, ribozyme-mediated RNA cleavage reaction. The Hammerhead ribozyme (HHR) can be engineered ligand-dependent by the attachment of an aptamer domain as a sensor unit to one of its stems. ${ }^{22,23}$ We have successively developed this regulation principle to function modularly in different species and RNA classes. In the beginning, utilization of an mRNA was controlled by the insertion of ligand-dependent ribozymes into eukaryotes and $E$. coli. ${ }^{8,24,25}$ In particular, we developed a
HHR-based artificial riboswitch in E. coli which depends on the accessibility of the ribosome binding site (RBS) (Fig. 1C). The HHR is attached to the mRNA in a way that only cleavage of the HHR leads to liberation of the RBS and hence results in translation of the message. ${ }^{24,26}$

In a further approach we demonstrated that ligand-dependent ribozymes can be designed in order to regulate tRNA activity in E. coli. For this purpose an amber suppressor tRNA ${ }^{\mathrm{Ser}}{ }_{\mathrm{CUA}}$ is rendered non-functional by joining of a HHR to the $5^{\prime}$-end of the tRNA. The disruption of the acceptor and D arms through base-pairing to an extension of the ribozyme is reversed by HHR-dependent cleavage and subsequent dissociation of the two fragments. The liberated tRNA can then suppress an amber stop codon on a reporter mRNA, thereby preventing premature termination of translation by the incorporation of serine. ${ }^{27}$

Importantly, our aptazymes have also been utilized to control siRNA processing ${ }^{28}$ and $16 \mathrm{~S}$ rRNA integrity. ${ }^{29}$ In addition, methods for the construction of orthogonal riboswitches with altered ligand specificities have been reported ${ }^{30-32}$ which make aptazymes highly suited for their modular assembly and the construction of multiple input computational devices. ${ }^{24,33}$ However, aptazyme-controlled functional RNAs have never been combined in a control network that performs higher-order computation in E. coli. Here, we show that the modular assembly of ligand-dependent RNAs allows the construction of genetic two-input logic gates. The control mechanisms rely on the catalytic activity of the HHRs since ligand-regulated cleavage results in either liberation of the RBS or the activation of a functional amber suppressor tRNA ${ }^{\mathrm{Ser}}$ CUA. 


\section{Results and discussion}

\section{Setup and expansion of the HHR-based toolbox}

In order to construct genetic two-input logic gates we designed an expression system where signals are integrated via two different HHR-based switches: the first controls translational initiation of an eGFP-coding mRNA and the other regulates amber stop codon suppression of the same message by regulating a cognate tRNA. The individual ribozymes are controlled by either the small molecule theophylline or the co-factor thiamine pyrophosphate (TPP), Fig. 1B and C.

The switches which we reported in earlier studies would have only allowed for the construction of a limited arsenal of binary logic gates. ${ }^{24,26,27}$ Therefore, we expanded and characterized the currently available collection. The screened aptazymes displayed high modularity because aptazymes screened within one context (in our case the tRNA system) allowed for the transfer to another functional RNA (the mRNA-based control of RBS) and retain their switching behaviour.

The construction of binary logic gates requires liganddependent "ON" and "OFF" switches. For example, the AND gate depends on the rewiring of two "ON" switches with diverging ligand-specificities (Fig. 1). The AND gate only gives rise to an binary output of 1 if both inputs are present but if either one or none of the inputs are present no binary output of 0 is produced. In contrast, the NOR gate can be realized by two "OFF" switches and an output of 1 is only produced if none of the inputs is present. Hence, the construction of various different biomolecular, gene-expression-based logic gates depends on a rich toolbox of "ON" and "OFF" switches with altered ligand specificities. However, the current toolbox $^{24,26,27}$ (Fig. 2A and B) would have only allowed for the creation of the AND and theo_ANDNOT_TPP gates because the assembly of the NOR gate and the TPP_ANDNOT_theo gate necessitates a TPP- or theophylline-dependent OFF-tRNA ${ }_{\text {CUA }}^{\text {Ser }}$ switch.

We expanded the collection of currently available HHR-based genetic switches within this study. We performed an in vivo screen for TPP-dependent ON-tRNA and OFF-tRNA switches. The identity of the connection sequence between the aptamer and the HHR scaffold has been proven to be crucial for the liganddependent switching behaviour of aptazymes. ${ }^{26}$ At first, the thiM aptamer domain ${ }^{12}$ was connected via a randomized connection sequence to the tRNA. Screening was performed in the presence and absence of thiamine in the growth medium and was based on differential expression of eGFP. Thiamine is actively transported into the cell and subsequently enzymatically converted into biologically active TPP. ${ }^{34}$ We were able to identify a TPPdependent ON-tRNA switch (Fig. 2C), whereas TPP-dependent OFF-tRNA switches were not obtained. This is in contrast to the previous screen in the context of mRNAs which yielded TPP-dependent ON-RBS as well as OFF-RBS switches. ${ }^{24}$

In addition, we screened for theophylline dependent OFF-tRNA switches. Screening of an asymmetric connection sequence of five randomized nucleotides resulted in a clone with OFF switch behaviour (Fig. 2C). We wondered whether the connection sequence of the OFF-tRNA switch allowed for the theophylline dependent sequestration of the RBS. Indeed, transfer of the screened aptazyme resulted in the generation of a theophylline-dependent OFF-RBS switch with a 2-fold reduced reporter gene expression at $2 \mathrm{mM}$ theophylline compared to the absence of an inducer in the culture medium. Hence, our results demonstrate the modular nature of aptazymes since a transfer from one functional RNA class to another by retaining their ligand-specific switching behaviour is facilitated. This observation is in accordance with previous studies by our group as well as Yokobayashi and co-workers. ${ }^{27-29}$

\section{Construction of two-input logic gates}

We next investigated whether two orthogonally acting riboswitches, one controlling the generation of a functional amber suppressor tRNA and the other controlling translation initiation of an mRNA (RBS), could be assembled in order to operate as binary logic gates (Fig. 1B and C). Importantly, all switches used in our study showed significantly altered reporter gene expression only in the presence of their assigned ligand with no cross-talk with the second ligand being observed (Fig. 2B and C). In addition, constitutive eGFP reporter gene expression was not influenced by any of the ligands (Fig. 3A). In general, we observed that combination of both tRNA and mRNA switches significantly reduced absolute reporter gene expression compared to systems with only tRNA or mRNA control mechanisms.

We first aimed for the creation of the AND gate (Fig. 3B). For this purpose, we combined the theoON-tRNA and the $\operatorname{tpp}_{1.20} \mathrm{ON}-\mathrm{RBS}$ switches to act on the expression of a single eGFP RNA transcript. Within this design only in the presence of TPP the RBS was accessible to the small ribosomal subunit as cleavage of the TPP-dependent HHR led to liberation of the SD sequence (Fig. 1C). However, if the second input theophylline is absent translation stops because the ribosome is unable to decode the amber stop codon. The presence of theophylline generates a functional tRNA ${ }^{\text {ser }}{ }_{\mathrm{CUA}}$ and results in production of a full-length fluorescent protein which corresponds to the binary output value of " 1 ". Indeed, highest eGFP expression was observed in the presence of both ligands, see Fig. 3B. The observed background eGFP expression presumably results from leaky control of the individual switches. As shown before, the switching performance of the individual switches is varying from 3- to 4-fold, see Fig. 2B. Upon combination, the residual expression in the "OFF" state adds up over both systems, resulting in higher background signals in the designed logic gates. By definition, binary logic gates display only two possible states ( 0 and 1$)$ and ideally gene expression should be either fully turned "ON" or "OFF" in genetically-encoded gates. However, perfect circuit performance is usually not achieved in reality due to a noisy environment and leaky control. ${ }^{35}$ Similarly, in electronic digital circuits signals are impaired by voltage drops and external factors. In order to cope with this phenomenon, the assignment of the binary digits 0 and 1 is based on the definition of a threshold value. Thereby, the analog response of the circuit is converted into a digital response discerning the " 0 " $/$ " 1 " or "ON"/ OFF" state. See below for a more detailed discussion about defining the threshold values.

In order to demonstrate the highly modular and orthogonal properties of the individual switches we then assembled an NOR gate (Fig. 3C). The NOR gate is of particular interest 

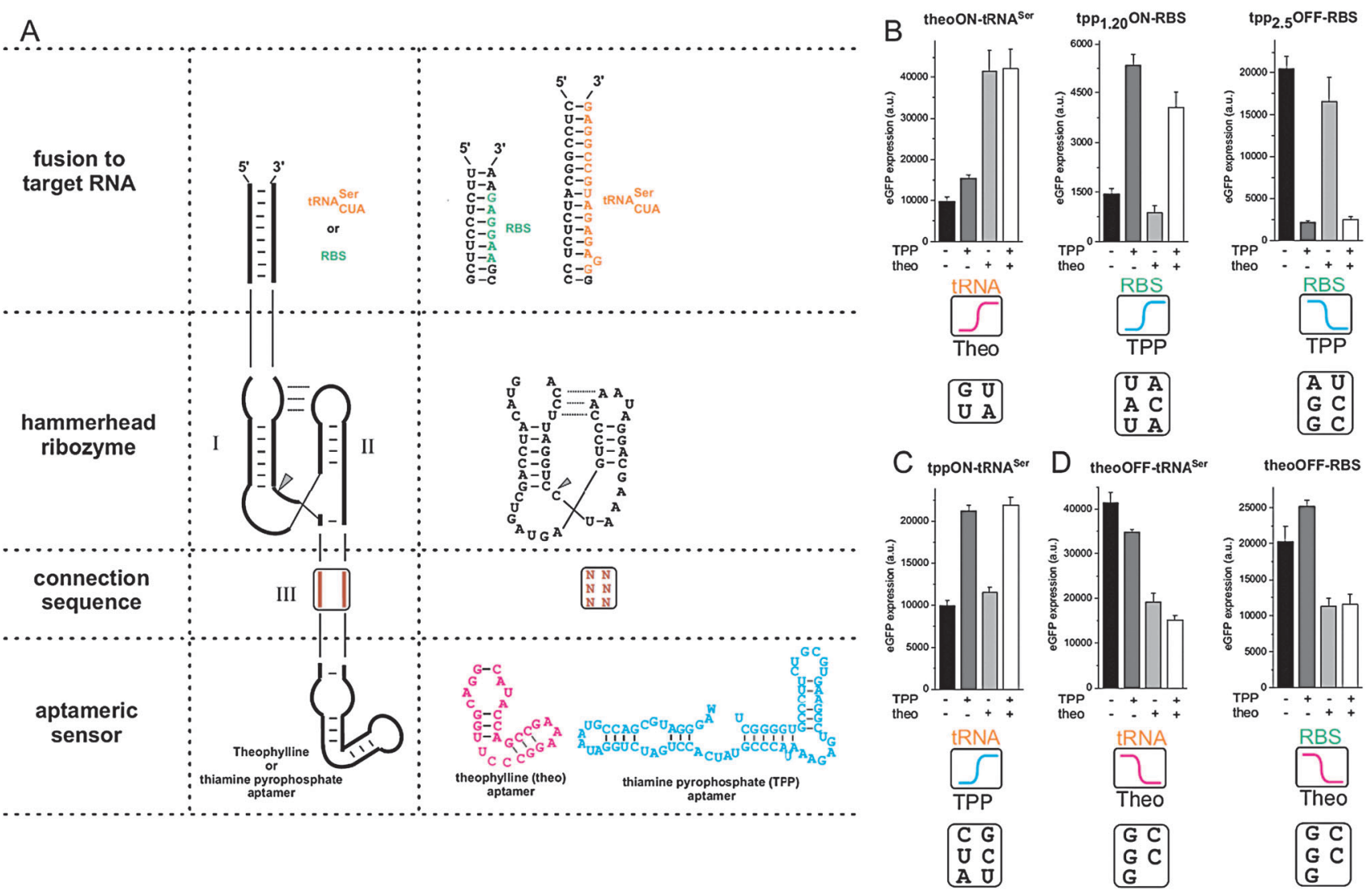

Fig. 2 Modular assembly of aptazymes and expansion of the HHR-switch toolbox. (A) A target RNA function (RBS within an mRNA or the amber suppressor tRNA) is joined to stem I of the HHR core. Allosteric HHRs are obtained by the joining of an aptameric sensor domain to stem III of the HHR. Screening of a pool of aptazymes with a randomized connection sequence allows for the identification of ligand-dependent aptazyme sequences. (B) Switches reported in previous studies were examined in the presence of thiamine ( $0.5 \mathrm{mM})$ or theophylline (2 mM). The nucleotide identity of the connection sequence for each switch is shown. Pictograms of switches indicate "ON" and "OFF" switches by positive and negative slopes. (C) and (D) Expansion of the HHR-switch toolbox by screening of libraries with randomized connection sequence resulted in the identification of tppON-tRNA ${ }^{\text {Ser }}$ and theoOFF-tRNA ${ }^{\text {Ser }}$ switches. (D) A theoOFF-RBS switch is generated by transferring the respective connection sequence from the theoOFF-tRNA ${ }^{\text {Ser }}$ aptazyme. (B) and (D) Experiments show eGFP fluorescence measured in triplicate in outgrown E. coli BL21 (DE3) gold strain grown in M63 minimal medium.

because it represents a universal building block for the construction of all other logic gates ${ }^{36}$ Implementation of the NOR gate was based on the assembly of the $\operatorname{tpp}_{1.20} \mathrm{OFF}$-RBS and theoOFF-tRNA ${ }^{\text {Ser }}$ switches. The NOR gate yielded high fluorescence values when thiamine and theophylline were absent in the culture medium; whereas the presence of either one or both inputs decreased gene expression by two fold.

The combination of "ON" and "OFF" switches with different ligand specificities resulted in ANDNOT logic operations (Fig. 3D to F). The ANDNOT gate discriminates between its inputs, and produces an output of 1 only if one input is present but the other input is absent. Hence, there are two possible ANDNOT gates with different switching behaviours, termed TPP_ANDNOT_theo and theo_ANDNOT_TPP in this study. In TPP_ANDNOT1_theo the tpp ${ }_{1.20} \mathrm{ON}-\mathrm{RBS}$ and theoOFF-tRNA ${ }^{\text {Ser }}$ switches were assembled which resulted in high fluorescence only if TPP was sensed. The same circuit behaviour was realized in ANDNOT3, in which theoOFFRBS and $\operatorname{tpp}_{1.20} \mathrm{ON}$-tRNA switches were combined. The theo_ANDNOT2_TPP operation was obtained in which only theophylline addition to the culture medium resulted in a binary output of " 1 " by combining the tpp ${ }_{2.5}$ OFF-RBS and
theoON-tRNA ${ }^{\text {Ser }}$ switches. Additional build-up of complexity could be obtained by using layers of switches which we reported in this study. For example the assembly of the TPP_ANDNOT_theo and theo_ANDNOT_TPP gates would allow for the construction of an XOR gate.

The gates which are presented in this study displayed a robust and reproducible switching behaviour. The greatest absolute changes in fluorescence are observed between the ON and the highest OFF state, whereas the changes between the individual OFF states were comparatively small. Consequentially, a wide range of potential threshold values of expressions are possible in order to define the respective logic behaviour. The individual threshold values are arbitrarily chosen for illustration purposes. By drastically shifting threshold values other logic behaviours are obtained, e.g. in Fig. 3E setting a threshold at 300 a.u. instead of 850 a.u. the former theo_ANDNOT_TPP gate would become a NOT gate. We have set threshold values for each constructed gate which in turn define the respective "ON" and "OFF" states of gene expression. In particular, the observed values for the "ON"/"OFF" ratios are comparable to a previous study, in which the construction of logic gates through cis-assembly of aptazymes resulted in switching 


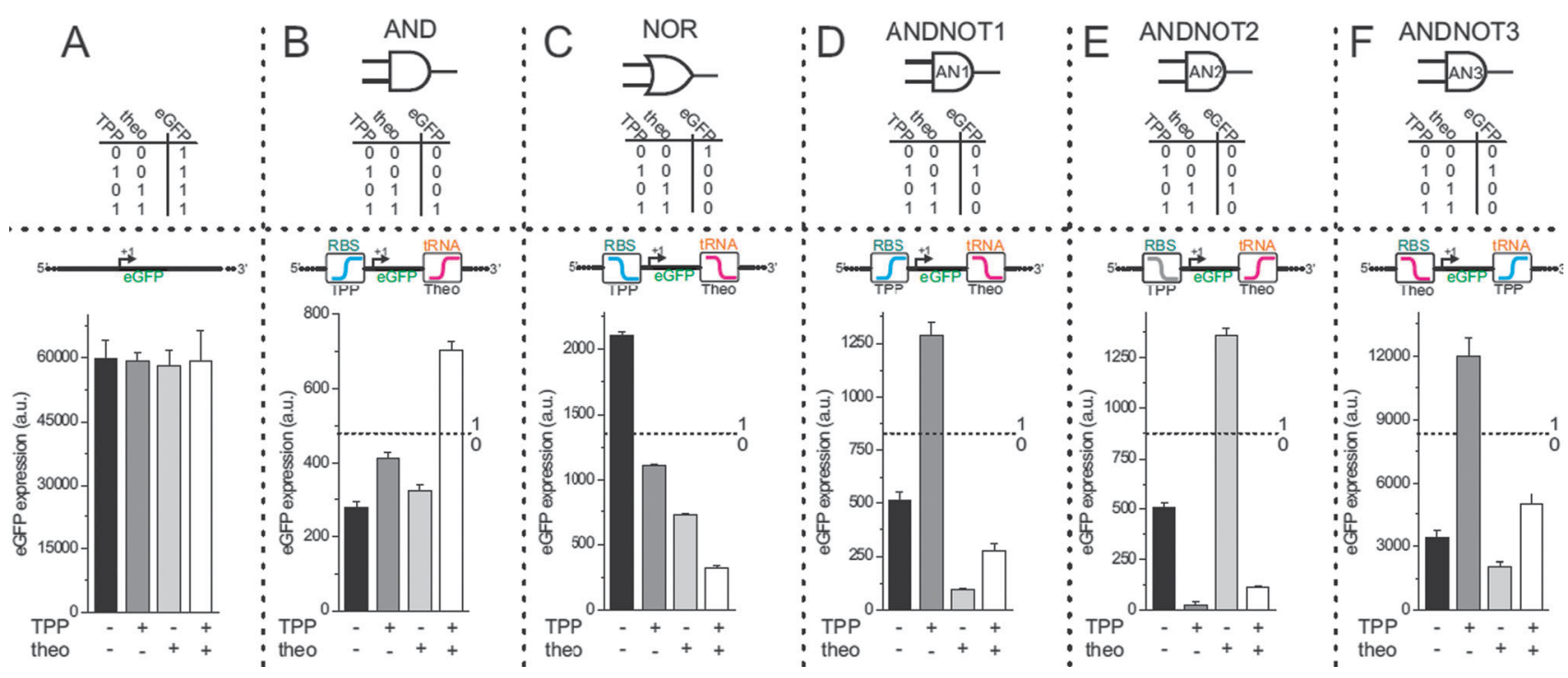

Fig. 3 Truth tables and performances of aptazyme-based two-input logic gates. (A) Addition of the respective inputs $(0.5 \mathrm{mM}$ thiamine; $2 \mathrm{mM}$ theophylline) into the culture medium had no influence on eGFP reporter gene expression alone. (B)-(F) Logic gates AND, NOR, TPP_ANDNOT_theo and theo_ANDNOT_TPP are shown. Pictograms within the mRNA identify the individual aptazymes utilized in order to construct the respective gates. A threshold value is indicated for each gate which discerned high (binary value " 1 ") from reduced (binary " 0 ") eGFP expression. Experiments show eGFP fluorescence measured in triplicate in outgrown E. coli BL21 (DE3) gold strain grown in M63 minimal medium.

ratios of 1.5 to 2.5 fold ${ }^{8,37} \mathrm{We}$ assume that the relatively high background fluorescence in the OFF state of our systems is primarily caused by leaky operation of the aptazyme-based switches.

For future applications the performance of the RNAswitches could be improved. In vivo evolution techniques and selection procedures are promising approaches for generating RNA regulators with even better performances. ${ }^{38,39}$ However, a recent example suggests that even small switching ratios of aptazyme-based regulatory devices are sufficient in order to control biological processes. ${ }^{40}$ In general, instead of using eGFP as a reporter gene for easy monitoring of the output expression more functional actuators could be expressed that interconnect with fundamental cellular processes such as intracellular responses or cell-to-cell communications. ${ }^{9,41,42}$ In the case of the tRNA riboswitches one source which contributes to a limited performance of these switches could be inefficient amber stop codon decoding because the amber suppressor tRNA competes with release factor 1 (RF1). In the context of genetic code expansion by incorporating unnatural amino acids RF1 knockout resulted in drastically increased efficiency of decoding. ${ }^{43,44}$

\section{Conclusion}

In general, the information processing capacity could be easily expanded if the presented RNA-based switches would be combined with protein-based strategies for conditional gene expression. ${ }^{14}$ This should open the possibility to construct more complex devices such as XOR, OR and NAND gates. Higher-order bio-computing devices in bacterial cells have so far only been established by encoding simple logic gates in distinct cell populations that are interconnected to consortia using quorum sensing. ${ }^{42}$ Increasing the circuit complexity in single host cells could be achieved by combining posttranscriptional RNA-based switches with transcriptional proteinbased strategies. In addition, the presented concept should not be restricted to $E$. coli. In previous studies by our group and others it was demonstrated that eukaryotic mRNA expression could be regulated by controlling mRNA stability in cis or by aptazyme-mediated pri-miRNA processing. ${ }^{8,45,46}$ Moreover, amber stop codon decoding has also been demonstrated in eukaryotes and aptazyme-dependent tRNA activation should be transferable to higher organisms as well. ${ }^{47,48}$ For example, the combination of various transcriptional- and translationalcontrol components resulted in programmable, complex circuits ranging from the XOR gate to the half-adder in single mammalian cells. ${ }^{49}$ This concept could also be applied for the genetic assembly of the half-adder and half-subtractor within one host bacterial cell and ultimately combined with cell-tocell communication to achieve even more complexity.

In summary, we rationally designed and constructed Boolean logic gates composed of two modular aptazyme-based RNA switches. Each of these switches responds to a different ligand input and controls a distinct RNA class. We expanded the currently available toolbox by several "ON" and "OFF" switches and demonstrated the modularity of the concept by transferring an aptazyme domain from one functional RNA class to another while retaining the switching behaviour. Combinations of the two control systems are achieved by both an aptazyme-mediated liberation of the ribosome binding site as well as activation of a tRNA that decodes an amber codon on a single eGFP transcript. Different gates such as the AND, NOR, and ANDNOT operation have been implemented with RNA switches displaying a variety of ligand-dependent activities. The results demonstrate that the utilized RNA switches are highly modular and can be assembled in an orthogonal fashion into more complex devices. Although the switching 
performances need to be optimized for even more complex assemblies, this and other studies demonstrate that RNA switches already represent versatile tools for engineering advanced genetic devices and circuits.

\section{Materials and methods}

\section{Plasmids for in vivo screening}

Two plasmid libraries, one containing theophylline aptamer and the other one containing the TPP-aptamer with randomized connection sequences, were created based on the previously reported template pMAB501. ${ }^{27}$ Plasmid libraries were generated by site directed mutagenesis using Phusion Polymerase (Finzyme). Primer pairs carried a $5^{\prime}$-overhang encoding the randomized connection sequence and the respective aptamer domain. For introduction of the theophylline-dependent pool the primers "pMAB 501 theoHHAz $3 \mathrm{~N}$ fw" and "pMAB 501 theoHHAz $2 \mathrm{~N}$ ry" and for the TPP-dependent pool the primers "pMAB 501 thiaHHAz $3 \mathrm{~N}$ fw" and "pMAB 501 thiaHHAz $3 \mathrm{~N}$ rv" were used. The template plasmid was digested with Dpn1 and the PCR products blunt-end ligated (Quick Ligase, NEB). Subsequently, the plasmid pool was transformed into E. coli BL21 (DE3) gold (Stratagene).

\section{Plasmid construction for two-input logic gates}

The plasmids encoding the two-input logic gates are based on the pMAB501 vector. ${ }^{27}$ All plasmids have a chloramphenicol acetyl transferase selection marker which was introduced by excision of the AmpR of pMAB501 using the primers "pHWB1 w/o ampRrv" and "pHWB1 w/o ampRfw", and insertion of the $C m R$ gene which was amplified from the $\mathrm{pMyr}$ plasmid with primers "pMyrchlorRfw" and "pMyrchlorRrv". An exception is the ANDNOT3 gate which was encoded on the original pMAB501 gate and carries the AmpR gene. For the construction of plasmids encoding two-input logic gates the respective RBS-dependent aptazymes were inserted into the $5^{\prime}$-UTR of eGFP within the pMAB501 plasmid by PCR with primers encoding the aptazyme sequence within their $5^{\prime}$ overhang. Successful molecular cloning of all constructed plasmids was confirmed by sequencing.

\section{Oligonucleotides used for $\mathrm{PCR}$ reactions}

pMAB 501 theoHHAz 3N fw GGCCCTTGGCAGGNNNT CCTGGATTCCAGGGAGAGATGCC

pMAB 501 theoHHAz 2N rv TTTCGGCTGGTATGNNT TTCGTCCTATTTGGGACTCATC

pMAB 501 thiaHHAz 3N fw GTATCACCTGATCTGGA TAATGCCAGCGTAGGGAANNNTCCTGGATTCCAGGG AGAGATGCC

pMAB 501 thiaHHAz 3N rv GGGTATTTCTCAGCCTT CACGCAGAAGGGCACCCCGANNNTTTCGTCCTATTT GGGACTCATC

RBS-onTPP-rev CCTTCACGCAGAAGGGCACCCCGATG TTTTCGTCCTATTTGGGACTCATCAGCTGGATGTACCG AAGGAGAAAGGGGAATTGTTATCCGCTCAC RBS-onTPP-for pCTGAGAAATACCCGTATCACCTGAT CTGGATAATGCCAGCGTAGGGATTATTCCTGGATTC CACGAAGGAGATATACCATGGGCCATCATC
RBS-offTPP-rev CCTTCACGCAGAAGGGCACCCCGA GGATTTCGTCCTATTTGGGACTCATCAGCTGGATGT ACCGAAGGAGAAAGGGGAATTGTTATCCGCTCAC

RBS-offTPP-for pCTGAgAAATACCCGTATCACCTGAT CTGGATAATGCCAGCGTAGGGATGGATCCTGGATTC CACGAAGGAGATATACCATGGGCCATCATC RBS-offTheo-rev TTCGGCTGGTATGGGTTTCGTCCTA TTTGGGACTCATCAGCTGGATGTACCGAAGGAGAAA GGGGAATTGTTATCCGCTCAC

RBS-offTheo-for AGGCCCTTGGCAGGGGGTCCTGGA TTCCACGAAGGAGATATACCATGGGCCATCATC pMyrchlorRfw p-TTACGCCCCGCCCTGCCAC pMyrchlorRrv p-ATGGAGAAAAAAATCACTGGATAT ACC

pHWB1 w/o ampRrv ACTCTTCCTTTTTCAATATTATT GAAGC

pHWB1 w/o ampRfw CTGTCAGACCAAGTTTACTC

$N$ represents an unbiased random position generated during solid phase DNA synthesis using a $1: 1: 1: 1$ mixture of nucleoside phosphoramidites. W constitutes $\mathrm{A}$ in the case of the tppONtRNA switch and $\mathrm{U}$ for the tppRBS switches.

\section{E. coli strains and media}

All experiments were conducted with the Escherichia coli "BL21 (DE3) gold" strain (Stratagene; $F^{-}$ompT gal dcm lon hsd $S_{\mathrm{B}}\left(r_{\mathrm{B}}{ }^{-} m_{\mathrm{B}}{ }^{-}\right) \lambda($ DE3 [lacI lacUV5-T7 gene 1 ind 1 sam 7 nin 5$\left.\left.]\right)\right)$. Bacterial cultures were grown in the respective medium, either Luria-Bertani (LB) medium or M63 minimal medium $\left(5 \mathrm{~g} \mathrm{~L}^{-1}\right.$ glucose, $2 \mathrm{mM} \mathrm{MgSO} 4,40 \mu \mathrm{g} \mathrm{L}^{-1}$ L-threonine, $40 \mu \mathrm{g} \mathrm{L}^{-1}$ methionine, $2 \mathrm{~g} \mathrm{~L}^{-1}\left(\mathrm{NH}_{4}\right)_{2} \mathrm{SO}_{4}, 13.6 \mathrm{~g} \mathrm{~L}^{-1} \mathrm{KH}_{2} \mathrm{PO}_{4}$, $0.5 \mathrm{mg} \mathrm{L}^{-1} \mathrm{FeSO}_{4}, 100 \mu \mathrm{g} \mathrm{L}^{-1}$ cyanocobalamine, $80 \mu \mathrm{g} \mathrm{L}^{-1}$ p-aminobenzoic acid, $20 \mu \mathrm{g} \mathrm{L}^{-1}$ biotin, $200 \mu \mathrm{g} \mathrm{L} \mathrm{L}^{-1}$ nicotinic acid, $100 \mu \mathrm{g} \mathrm{L}{ }^{-1} \mathrm{Ca}-\mathrm{D}(+)$-pantothenate, $300 \mu \mathrm{g} \mathrm{L}^{-1}$ pyridoxamine chloride) supplemented with either $100 \mu \mathrm{g} \mathrm{mL}^{-1}$ carbenicillin or $34 \mu \mathrm{g} \mathrm{mL}^{-1}$ chloramphenicol.

\section{In vivo screening}

In general, single colonies were picked into medium and incubated vigorously shaking at $37^{\circ} \mathrm{C}$. After grown-out, cells were regrown in the presence and absence of the respective inducers and the GFP fluorescence was determined. Initial screening was performed in 384-well plates and hits were validated as triplicates in 96-well plates. The screening of theophylline-dependent switches was performed in LB medium, whereas screening of TPP-dependent switches was carried out in M63 medium. In order to obtain the identity of the randomized nucleotides, plasmids were isolated (Miniprep Kit, Zymo Research) and sequenced.

\section{Two-input logic gates}

Cells were grown overnight in M63 medium supplemented with antibiotics. Bacterial suspensions were then diluted 1:100 into fresh M63 medium containing the respective inducers ( $2 \mathrm{mM}$ theophylline and/or $500 \mu \mathrm{M}$ thiamine) and grown at $37{ }^{\circ} \mathrm{C}$ vigorously shaking overnight.

\section{eGFP fluorescence measurements}

$100 \mu \mathrm{L}$ of an outgrown culture was transferred into a 96-well microtiter plate. For 384-well plates $50 \mu \mathrm{L}$ of bacterial 
suspension was used. The fluorescence of the expressed eGFP was measured with a Tecan M200 plate reader (excitation wavelength $=488 \mathrm{~nm}$, emission wavelength $=535 \mathrm{~nm}$ ). Fluorescence of an equally treated culture not expressing eGFP was determined and used for background subtraction.

\section{Notes and references}

1 W. Weber and M. Fussenegger, Nat. Rev. Genet., 2012, 13, 21-35.

2 M. B. Elowitz and S. Leibler, Nature, 2000, 403, 335-338.

3 A. E. Friedland, T. K. Lu, X. Wang, D. Shi, G. Church and J. J. Collins, Science, 2009, 324, 1199-1202.

4 W. Weber, J. Stelling, M. Rimann, B. Keller, M. Daoud-El Baba, C. C. Weber, D. Aubel and M. Fussenegger, Proc. Natl. Acad. Sci. U. S. A., 2007, 104, 2643-2648.

5 T. S. Gardner, C. R. Cantor and J. J. Collins, Nature, 2000, 403, 339-342.

6 A. Levskaya, A. A. Chevalier, J. J. Tabor, Z. B. Simpson, L. A. Lavery, M. Levy, E. A. Davidson, A. Scouras, A. D. Ellington, E. M. Marcotte and C. A. Voigt, Nature, 2005, 438, 441-442.

7 K. Rinaudo, L. Bleris, R. Maddamsetti, S. Subramanian, R. Weiss and Y. Benenson, Nat. Biotechnol., 2007, 25, 795-801.

8 M. N. Win and C. D. Smolke, Science, 2008, 322, 456-460.

9 A. Tamsir, J. J. Tabor and C. A. Voigt, Nature, 2011, 469, 212-215.

10 C. Guerrier-Takada, K. Gardiner, T. Marsh, N. Pace and S. Altman, Cell, 1983, 35, 849-857.

11 T. R. Cech, A. J. Zaug and P. J. Grabowski, Cell, 1981, 27, 487-496.

12 W. Winkler, A. Nahvi and R. R. Breaker, Nature, 2002, 419, 952-956.

13 D. Auslander, M. Wieland, S. Auslander, M. Tigges and M. Fussenegger, Nucleic Acids Res., 2011, 39, e155.

14 A. de Las Heras, C. A. Carreno, E. Martinez-Garcia and V. de Lorenzo, FEMS Microbiol. Rev., 2010, 34, 842-865.

15 N. E. Buchler, U. Gerland and T. Hwa, Proc. Natl. Acad. Sci. U. S. A., 2003, 100, 5136-5141.

16 M. N. Stojanovic and D. Stefanovic, J. Am. Chem. Soc., 2003, 125, 6673-6676.

17 A. Nahvi, N. Sudarsan, M. S. Ebert, X. Zou, K. L. Brown and R. R. Breaker, Chem. Biol., 2002, 9, 1043.

18 A. Roth and R. R. Breaker, Annu. Rev. Biochem., 2009, 78, 305-334.

19 E. R. Lee, J. L. Baker, Z. Weinberg, N. Sudarsan and R. R. Breaker, Science, 2010, 329, 845-848.

20 C. D. Stoddard and R. T. Batey, ACS Chem. Biol., 2006, 1, 751-754.

21 N. Sudarsan, M. C. Hammond, K. F. Block, R. Welz, J. E. Barrick, A. Roth and R. R. Breaker, Science, 2006, 314, 300-304.

22 J. Tang and R. R. Breaker, Chem. Biol., 1997, 4, 453-459.

23 M. Wieland and J. S. Hartig, ChemBioChem, 2008, 9, 1873-1878.

24 M. Wieland, A. Benz, B. Klauser and J. S. Hartig, Angew. Chem., Int. Ed., 2009, 48, 2715-2718.
25 I. T. Seemann and J. S. Hartig, Synlett, 2011, 1486-1494.

26 M. Wieland and J. S. Hartig, Angew. Chem., Int. Ed., 2008, 47, 2604-2607.

27 B. Berschneider, M. Wieland, M. Rubini and J. S. Hartig, Angew. Chem., Int. Ed., 2009, 48, 7564-7567.

28 D. Kumar, C. I. An and Y. Yokobayashi, J. Am. Chem. Soc., 2009, 131, 13906-13907.

29 M. Wieland, B. Berschneider, M. D. Erlacher and J. S. Hartig, Chem. Biol. (Cambridge, MA, U. S.), 2010, 17, 236-242.

30 A. Saragliadis, B. Klauser and J. S. Hartig, Methods Mol. Biol., 2012, 848, 455-463.

31 N. Dixon, J. N. Duncan, T. Geerlings, M. S. Dunstan, J. E. McCarthy, D. Leys and J. Micklefield, Proc. Natl. Acad. Sci. U. S. A., 2010, 107, 2830-2835.

32 N. Dixon, C. J. Robinson, T. Geerlings, J. N. Duncan, S. P. Drummond and J. Micklefield, Angew. Chem., Int. Ed., 2012, 51, 3620-3624.

33 M. Wieland, M. Gfell and J. S. Hartig, $R N A$, 2009, 15, 968-976.

34 C. T. Jurgenson, T. P. Begley and S. E. Ealick, Annu. Rev. Biochem., 2009, 78, 569-603.

35 M. B. Elowitz, A. J. Levine, E. D. Siggia and P. S. Swain, Science, 2002, 297, 1183-1186.

36 C. S. Peirce, Collected Papers of Charles Sanders Peirce, Harvard University Press, Cambridge, 1931-1935.

37 X. Chen and A. D. Ellington, PLoS Comput. Biol., 2009, 5, e1000620.

38 Y. Nomura and Y. Yokobayashi, J. Am. Chem. Soc., 2007, 129, 13814-13815.

39 K. M. Esvelt, J. C. Carlson and D. R. Liu, Nature, 2011, 472, 499-503.

40 Y. Y. Chen, M. C. Jensen and C. D. Smolke, Proc. Natl. Acad. Sci. U. S. A., 2010, 107, 8531-8536.

41 S. J. Culler, K. G. Hoff and C. D. Smolke, Science, 2010, 330, 1251-1255.

42 S. Regot, J. Macia, N. Conde, K. Furukawa, J. Kjellen, T. Peeters, S. Hohmann, E. de Nadal, F. Posas and R. Sole, Nature, 2011, 469, 207-211.

43 D. B. Johnson, J. Xu, Z. Shen, J. K. Takimoto, M. D. Schultz, R. J. Schmitz, Z. Xiang, J. R. Ecker, S. P. Briggs and L. Wang, Nat. Chem. Biol., 2011, 7, 779-786.

44 T. Mukai, A. Hayashi, F. Iraha, A. Sato, K. Ohtake, S. Yokoyama and K. Sakamoto, Nucleic Acids Res., 2010, 38, 8188-8195.

45 S. Auslander, P. Ketzer and J. S. Hartig, Mol. BioSyst., 2010, 6 , 807-814.

46 L. Yen, J. Svendsen, J. S. Lee, J. T. Gray, M. Magnier, T. Baba, R. J. D'Amato and R. C. Mulligan, Nature, 2004, 431, 471-476.

47 C. C. Liu and P. G. Schultz, Annu. Rev. Biochem., 2010, 79, 413-444.

48 J. W. Chin, T. A. Cropp, J. C. Anderson, M. Mukherji, Z. Zhang and P. G. Schultz, Science, 2003, 301, 964-967.

49 S. Ausländer, D. Ausländer, M. Müller, M. Wieland and M. Fussenegger, Nature, 2012, DOI: 10.1038/nature11149 [Epub ahead of print]. 